\title{
Influência da cirurgia bariátrica no tratamento com implantes dentários
}

\author{
Influence of bariatric surgery on dental implant treatment \\ Influencia de la cirugía bariátrica en el tratamiento con implantes dentales
}

Ana Viviam Souza Ferro Gomes ORCID: https://orcid.org/0000-0003-3441-8205 Centro Universitário Dom Bosco, Brasil E-mail: anavivianfg@gmail.com

Maria Clara de Sena Vieira

ORCID: https://orcid.org/0000-0002-7092-8950 Centro Universitário Dom Bosco, Brasil E-mail: mariaclaras1999@ hotmail.com Lara Carolyne de Sousa Flor ORCID: https://orcid.org/0000-0002-5525-4572 Centro Universitário Dom Bosco, Brasil

E-mail: laracarolynef@gmail.com

Luana Barbieri Trinta

ORCID: https://orcid.org/0000-0002-5625-3299 Centro Universitário Dom Bosco, Brasil E-mail: academico.barbieri@gmail.com

Anna Carolina Azevedo Sousa

ORCID: https://orcid.org/0000-0002-9849-4648 Centro Universitário Dom Bosco, Brasil

E-mail: carol_as@hotmail.com

Ana Cláudia Caldas Santos

ORCID: https://orcid.org/0000-0001-5676-0023 Centro Universitário Dom Bosco, Brasil E-mail: ana.claudia_nina@hotmail.com Yngrid Beatriz Silva Coelho

ORCID: https://orcid.org/0000-0002-6366-3069 Centro Universitário Dom Bosco, Brasil

E-mail: ynbeatriz@outlook.com

Emiliana Tereza Costa Lindoso

ORCID: https://orcid.org/0000-0002-9393-4448 Centro Universitário Dom Bosco, Brasil

E-mail: emiliana_002@hotmail.com

Lyandra Estephane Costa Mousinho

ORCID: https://orcid.org/0000-0002-6107-8333 Centro Universitário Dom Bosco, Brasil E-mail: lyandra.estephane@hotmail.com

Sinara Marques Campos

ORCID: https://orcid.org/0000-0002-2996-6453 Centro Universitário Dom Bosco, Brasil E-mail: sinaramarqs_19@hotmail.com

Janeth Corrêa Almeida

ORCID: https://orcid.org/0000-0003-4944-1061 Centro Universitário Dom Bosco, Brasil E-mail: janethalmeida212@gmail.com

Claudio Vanucci Silva de Freitas ORCID: https://orcid.org/0000-0003-0492-8423 Centro Universitário Dom Bosco, Brasil E-mail: claudiovanucci@hotmail.com

Maurício Silva Demétrio

ORCID: https://orcid.org/0000-0002-4855-370X Centro Universitário Dom Bosco, Brasil

E-mail: cirurgia@mauriciodemetrio.com.br

José Manuel Noguera Bazán

ORCID: https://orcid.org/0000-0002-1700-8995 Centro Universitário Dom Bosco, Brasil

E-mail: jmnbazan@hotmail.com

Cícero Newton Lemos Felicio Agostinho

ORCID: https://orcid.org/0000-0002-5658-3749

Centro Universitário Dom Bosco, Brasil

E-mail: ciceronewton@yahoo.com.br 


\title{
Resumo
}

A obesidade afeta o organismo e torna propício para o surgimento de diabetes, hipertensão arterial e até potencializar inflamação periodontal ou peri-implantar. Nesse cenário, como tratamentos convencionais há mudança alimentar, exercício físico, medicação e entre outras formas. Caso não obtenha resultado favorável, indica-se cirurgia bariátrica que auxilia na redução de peso, sendo mais utilizada a técnica bypass gástrico em Y-de-Roux. Após gastroplastia, pode ocorrer deficiência nutricional capaz de impactar na estruturação óssea, dentária, periodontal e salivar. O objetivo principal da revisão de literatura foi verificar se o déficit nutricional em paciente submetido à cirurgia bariátrica pode afetar o tratamento com implantes dentários. A busca foi realizada nas plataformas Scielo, Google Acadêmico e PubMed, nos idiomas português e inglês, entre 2010 e 2021, com as palavras-chaves: "cirurgia bariátrica", "implante dentário", "deficiências nutricionais" e "obesidade" e suas respectivas versões no idioma inglês. No total, 28 publicações foram obtidas, sendo 13 selecionadas a partir dos critérios de inclusão. Os estudos mostram associação da gastroplastia com erosão dental, cárie, doenças periodontais, xerostomia e deficiências nutricionais, sobretudo de cálcio e vitamina $\mathrm{D}$, que estão associadas a osteoporose e hiperparatireoidismo secundário e podem afetar negativamente o metabolismo e estrutura óssea e tratamento com implantes dentários devido à perda óssea pela maior ação de osteoclastos e possibilidade de inflamação peri-implantar. Entretanto, não há evidências científicas suficientes para afirmar que a deficiência nutricional ocasionada pela gastroplastia é capaz de reduzir o potencial de osseointegração ou a longevidade dos implantes. Mais estudos são necessários para esclarecer essa relação.

Palavras-chave: Cirurgia bariátrica; Implante dentário; Deficiências Nutricionais; Obesidade.

\begin{abstract}
Obesity affects the body and makes it conducive to the onset of diabetes, high blood pressure and even potentiate periodontal or peri-implant inflammation. In this scenario, as conventional treatments, there is food change, physical exercise, medication and among other forms. If a favorable result is not obtained, bariatric surgery is recommended to help with weight reduction, with the Roux-en-Y gastric bypass technique being more used. After gastroplasty, nutritional deficiency can occur that can impact bone, dental, periodontal and salivary structure. The main objective of the literature review was to verify whether the nutritional deficit in a patient undergoing bariatric surgery can affect treatment with dental implants. The search was carried out on the Scielo, Google Scholar and PubMed platforms, in Portuguese and English, between 2010 and 2021, with the keywords: "bariatric surgery", "dental implant", "nutritional deficiencies" and "obesity" and their English language versions. In total, 28 publications were obtained, 13 of which were selected based on the inclusion criteria. Studies show an association of gastroplasty with dental erosion, caries, periodontal diseases, dry mouth and nutritional deficiencies, especially calcium and vitamin $\mathrm{D}$, which are associated with osteoporosis and secondary hyperparathyroidism and can negatively affect the metabolism and bone structure and treatment with dental implants due to bone loss due to the greater action of osteoclasts and the possibility of peri-implant inflammation. However, there is insufficient scientific evidence to affirm that the nutritional deficiency caused by gastroplasty is able to reduce the potential for osseointegration or the longevity of the implants. Further studies are needed to clarify this relationship.
\end{abstract}

Keywords: Bariatric surgery; Dental implant; Nutritional deficiencies; Obesity.

\section{Resumen}

La obesidad afecta o el cuerpo se vuelve propenso a la aparición de diabetes, hipertensión arterial y potencia la inflamación periodontal o periimplantaria. En este escenario, al igual que los tratamientos convencionales, tiene un cambio de alimentación, ejercicio, medicación y otras formas. Si no se obtiene un resultado favorable, está indicada la cirugía bariátrica, que ayuda en la reducción de peso, siendo más utilizada la técnica de bypass gástrico en Y de Roux. La gastroplastia puede provocar una deficiencia nutricional capaz de afectar estructuras óseas, dentales, periodontales y salivales. El principal objetivo de la revisión de la literatura fue verificar si el déficit nutricional en un paciente sometido a cirugía bariátrica puede afectar o tratar con implantes dentales. Se realizó una búsqueda en las plataformas Scielo, Google Academic y PubMed, en portugués e inglés, de 2010 a 2021, con las palabras: "cirugía bariátrica", "implante dental", "deficiencias nutricionales" y "obesidad" y sus respectivos no - Versiones en inglés. En total se obtuvieron 28 publicaciones, 13 de las cuales se seleccionaron en base a dos criterios de inclusión. Los estudios muestran una asociación de la gastroplastia con erosión dental, cáncer, enfermedades periodontales, xerostomía y deficiencias nutricionales, exceso de calcio y vitamina $\mathrm{D}$, que se asocian con osteoporosis e hiperparatiroidismo secundario y pueden afectar negativamente el metabolismo y la estructura ósea del tratamiento óseo y los implantes. más osteoclastos se deshacen del hueso y existe la posibilidad de inflamación periimplantaria. Mientras tanto, no hay suficiente evidencia científica para afirmar que la deficiencia nutricional causada por la gastroplastia sea capaz de reducir el potencial de osteointegración o la longevidad de dos implantes. Se necesitan más estudios para aclarar esta relación.

Palabras clave: Cirugía bariátrica; Implante dental; Deficiencias nutricionales; Obesidad. 


\section{Introdução}

A obesidade é uma doença multifatorial, crônica e não infecciosa, que envolve aspectos hormonais, genéticos, comportamentais, ambientais, sedentarismo e ingestão de alimentos de grande densidade, sendo caracterizada pelo acúmulo excessivo de gordura no corpo. Essa patologia pode afetar o sistema cardiovascular, respiratório, nervoso e até mesmo contribuir para o desenvolvimento de diabetes, hipertensão arterial, câncer e entre outras enfermidades (Cândido, et al., 2021).

Assim, em caso de obesidade grave do tipo mórbida, paciente com índice de massa corporal (IMC) $35-40 \mathrm{~kg} / \mathrm{m}^{2}$, associada a algum comprometimento/complicação clínica e/ou que não obteve êxito com tratamentos convencionais, indica-se à cirurgia bariátrica, que favorece a perda de peso

Como possíveis consequências, no pós-cirúrgico, destacam-se as deficiências nutricionais capazes de influenciar à cavidade bucal, afetando dentes, ossos e saliva. Dentro desse contexto, com a absorção dificultada de cálcio ativo e vitamina D, o metabolismo ósseo pode ser afetado a longo prazo, favorecendo o desenvolvimento de doenças ósseas como a osteoporose (Santos et al., 2019). Nessa perspectiva, é crucial que o implantodontista conheça o estado de saúde-doença de seu paciente, a partir de análise pré-operatória, por meio de anamnese, exame físico-clínico e complementares, com finalidade de direcionar assertivamente o caso (Martins et al., 2011). Conforme esse cenário, o objetivo principal da revisão de literatura foi verificar se o déficit nutricional em pacientes submetidos à cirurgia bariátrica pode influenciar o tratamento com implantes dentários.

\section{Metodologia}

Esta revisão de literatura analisou trabalhos científicos publicados nas bases de dados Google Acadêmico, PubMed e BBO, publicados entre os anos de 2010 e 2021, nos idiomas português e inglês. Os descritores utilizados para busca foram: "Cirurgia Bariátrica", "Implante dentário" e posteriormente incluídos "Deficiências Nutricionais" e "Obesidade". 
Fluxograma 1.

Estudos identificados por meio de pesquisa em bases de dados $(n=145)$, Google Acadêmico $(n=143)$, Pubmed ( $\mathrm{n}=1)$ e BBO $(\mathrm{n}=1)$.

Estudos removidos por duplicatas e sem livre acesso $(\mathrm{n}=30)$
Estudos selecionados $(\mathrm{n}=55)$

Estudos selecionados para leitura completa $(n=25)$
Estudos removidos por fuga ao tema proposto $(\mathrm{n}=10)$

Fonte: Autores.

Foram incluídos revisões de literatura sistemáticas e trabalhos que abordassem as repercussões clínicas após cirurgia bariátrica e sua possível relação com a saúde bucal e implantes dentários; além da importância da avaliação clínica do paciente antes de tratamento com implantes dentários. De acordo com Munzlinger et al. (2012) a revisão sistemática consiste em um processo de pesquisa baseado em estudos bibliográficos, objetivando proceder ao levantamento formal. 


\section{Quadro 1. Artigos selecionados.}

\begin{tabular}{|c|c|c|}
\hline Autores e ano & Objetivo & Conclusão \\
\hline $\begin{array}{l}\text { Alasqah; Al-Shibani; Al-Aali; Qutub; } \\
\text { Abduljabbar; Akram (2019). }\end{array}$ & $\begin{array}{l}\text { Estimar e comparar os índices clínicos e } \\
\text { radiográficos e os níveis PICF de IL-1 } \beta \text { e IL-6 } \\
\text { entre pacientes obesos e não obesos }\end{array}$ & $\begin{array}{l}\text { Os achados do presente estudo sugerem que o aumento de } \\
\text { biomarcadores proinflamatórios no PICF de pacientes } \\
\text { obesos pode modular a inflamação peri-implante em torno } \\
\text { de implantes dentários. }\end{array}$ \\
\hline Bubola et al. (2018) & $\begin{array}{l}\text { Abordar a importância da vitamina } \mathrm{D} \text { e o efeito } \\
\text { de sua deficiência sobre a saúde sistêmica e a } \\
\text { repercussão na cavidade bucal com implantes } \\
\text { dentários. }\end{array}$ & $\begin{array}{l}\text { São necessárias mais pesquisas que possam elucidar } \\
\text { melhor os mecanismos de ação da vitamina } D \text { nos } \\
\text { processos de metabolismo ósseo, da osseointegração e no } \\
\text { sucesso e sobrevida dos implantes }\end{array}$ \\
\hline Cabral et al. (2016) & $\begin{array}{l}\text { Avaliar o impacto da deficiência de vitamina D } \\
\text { e do cálcio ósseo de pacientes após } \\
\text { gastroplastia em Y-de-Roux, relacionando tipo } \\
\text { de administração, dose e efeito pós-cirúrgico. }\end{array}$ & $\begin{array}{l}\text { Os pacientes submetidos ao RYGB devem fazer uso de } \\
\text { polivitamínicos e minerais principalmente a vitamina D e o } \\
\text { cálcio para evitar fraturas ósseas. }\end{array}$ \\
\hline $\begin{array}{l}\text { Cândido; Moreira; } \quad \text { Alexandre; } \\
\text { Cavalcante (2021). }\end{array}$ & $\begin{array}{l}\text { Relato de caso com análise documental por } \\
\text { meio da revisão do prontuário, relatórios de } \\
\text { evolução médica e de enfermagem, exames } \\
\text { laboratoriais e Tomografia Computadorizada, } \\
\text { durante o período de internamento do paciente } \\
\text { com obesidade em um hospital público. }\end{array}$ & $\begin{array}{l}\text { Percebeu-se que tanto na obesidade como na COVID-19 os } \\
\text { processos inflamatórios são comuns e evidenciados pelos } \\
\text { mesmos marcadores; esses processos podem exacerbar a } \\
\text { infecção causada pelo } \\
\text { SARS-CoV2. }\end{array}$ \\
\hline Souza; Oliveira; Lages; Fernandes (2018) & $\begin{array}{l}\text { Investigar o efeito da cirurgia bariátrica no } \\
\text { estado periodontal através de uma revisão } \\
\text { sistemática. }\end{array}$ & $\begin{array}{l}\text { O índice da placa pode ser melhorado após a cirurgia } \\
\text { bariátrica. }\end{array}$ \\
\hline Hazem; Bissada; Demko; Paes; Lang (2016) & $\begin{array}{l}\text { Comparar a frequência de falha do implante e } \\
\text { complicações entre pacientes obesos e } \\
\text { nonobese. }\end{array}$ & $\begin{array}{l}\text { A categoria IMC esteve associada a complicações do } \\
\text { implante; pacientes obesos têm maiores chances de } \\
\text { experimentar complicações de implantes pós-cirurgia. }\end{array}$ \\
\hline $\begin{array}{l}\text { Lefebvre; Letois; Sultan; Nocca; Mura; Galtier } \\
\text { (2014) }\end{array}$ & $\begin{array}{l}\text { Avaliar diversas vitaminas, nutrientes e } \\
\text { marcadores nutricionais e seus determinantes } \\
\text { em pacientes com obesidade considerando a } \\
\text { cirurgia bariátrica. }\end{array}$ & $\begin{array}{l}\text { Verificou-se que o alto índice de massa corporal e alta } \\
\text { glicemia plasmática de jejum, aumenta o risco de } \\
\text { deficiências, particularmente para 25(OH)D. Deve-se } \\
\text { aconselhar a triagem pré-operatória e a correção de } \\
\text { deficiências. }\end{array}$ \\
\hline $\begin{array}{l}\text { Martins; Bonilha; Falcón-Antenucci; Verri; } \\
\text { Verri (2011) }\end{array}$ & $\begin{array}{l}\text { Realizar uma revisão da literatura analisando } \\
\text { esses fatores que podem influenciar na } \\
\text { osseointegração e determinar métodos que } \\
\text { levam ao sucesso clínico da osseointegração. }\end{array}$ & $\begin{array}{l}\text { Os fatores sistêmicos podem influenciar de forma } \\
\text { significativa no sucesso clínico dos implantes dentários e } \\
\text { seria ideal que o implantodontista sempre planejasse o } \\
\text { tratamento em conjunto com o protesista que irá finalizá-lo. }\end{array}$ \\
\hline $\begin{array}{l}\text { Monteiro; Pellizzer; } \quad \text { Lemos; } \quad \text { Moraes; } \\
\text { Vasconcelos (2019) }\end{array}$ & $\begin{array}{l}\text { Testar as seguintes hipóteses: } \\
\text { (1) que não há diferença na taxa de } \\
\text { sobrevivência do implante entre os indivíduos } \\
\text { com sobrepeso ou obesidade e aqueles que } \\
\text { estão dentro da faixa de peso ideal; (2) que não } \\
\text { há diferenças entre esses grupos em relação aos } \\
\text { indicadores de saúde peri-implantar. }\end{array}$ & $\begin{array}{l}\text { Quão-sempre, há alguma evidência de que estes pacientes } \\
\text { obesos podem estar em maior risco de doenças clínicas } \\
\text { complicações em comparação com aqueles que estão } \\
\text { dentro da faixa de peso ideal. }\end{array}$ \\
\hline $\begin{array}{l}\text { Mosquim; Junior; Hissano; Wang; Peres } \\
\text { (2019) }\end{array}$ & $\begin{array}{l}\text { Revisar a literatura acerca da relação entre } \\
\text { obesidade, cirurgia bariátrica e alterações } \\
\text { bucais. }\end{array}$ & $\begin{array}{l}\text { A literatura evidencia a importância do cirurgião-dentista } \\
\text { no controle de riscos, na prevenção e no tratamento dos } \\
\text { pacientes obesos e bariátricos. }\end{array}$ \\
\hline Moura-Grec et al. (2012) & $\begin{array}{l}\text { Identificar consequências desse procedimento e } \\
\text { suas repercussões na saúde bucal. }\end{array}$ & $\begin{array}{l}\text { A manutenção da saúde bucal adequada em pacientes } \\
\text { submetidos à cirurgia bariátrica contribui para o sucesso } \\
\text { após a operação, resguardando os benefícios e } \\
\text { minimizando os efeitos colaterais. }\end{array}$ \\
\hline Pinheiro (2019) & $\begin{array}{l}\text { Fazer uma avaliação histomorfométrica da } \\
\text { osseointegração de implantes dentários nesses } \\
\text { pacientes }\end{array}$ & $\begin{array}{l}\text { Não ocorreu diferença significativa entre os grupos } \\
\text { avaliados em relação a quantidade de tecido ósseo } \\
\text { formado. }\end{array}$ \\
\hline Quintella et al. (2020) & $\begin{array}{l}\text { Avaliar se a cirurgia bariátrica apresentava } \\
\text { maior risco de erosão dentária. }\end{array}$ & $\begin{array}{l}\text { Os pacientes submetidos à cirurgia bariátrica apresentaram } \\
\text { maior incidência de erosão dentária. }\end{array}$ \\
\hline Santos et al. (2019) & $\begin{array}{l}\text { Discutir a relação de alterações na cavidade } \\
\text { bucal como resultado da cirurgia bariátrica. }\end{array}$ & $\begin{array}{l}\text { É necessário que o cirurgião-dentista participe da equipe } \\
\text { multidisciplinar que acompanha os pacientes obesos que se } \\
\text { submeterão à cirurgia bariátrica, contribuindo para a } \\
\text { diminuição de efeitos bucais e aumento da qualidade de } \\
\text { vida. }\end{array}$ \\
\hline Vargas; Bonato; Orenha; Sales (2020) & $\begin{array}{l}\text { Verificar o padrão ósseo alveolar por meio de } \\
\text { índices radiomorfométricos da radiografia } \\
\text { panorâmica e medidas lineares realizadas em } \\
\text { radiografias periapicais, em pacientes } \\
\text { eutróficas e obesas mórbidas antes e após a } \\
\text { cirurgia bariátrica. }\end{array}$ & $\begin{array}{l}\text { Pacientes obesas apresentam maior perda óssea, a qual } \\
\text { piora após a operação bariátrica, quando comparada com } \\
\text { pacientes eutróficas. O mesmo acontece com o trabeculado } \\
\text { ósseo que se torna mais esparso após a operação. }\end{array}$ \\
\hline
\end{tabular}

Fonte: Autores. 


\section{Resultados e Discussão}

A obesidade é considerada um problema de saúde que traz consequências negativas ao organismo. Quanto à sua classificação, segundo a Organização Mundial de Saúde (OMS), pode ser: grau I, quando IMC é entre 30-34,9 Kg/m²; obesidade grau II 35-39,9 Kg/m² e obesidade grau III se IMC > a $40 \mathrm{Kg} / \mathrm{m}^{2}$ (Santos et al., 2019).

Às possibilidades de tratamento para casos de obesidade podem ser a reeducação alimentar, dieta hipocalórica, medicamentos e atividades físicas. Quando essas alternativas não são suficientes para tratar a obesidade, especialmente a de grau III, indica-se a cirurgia bariátrica, também conhecida como gastroplastia ou cirurgia da obesidade/redução de estômago. Tal procedimento gera modificações na anatomia e fisiologia do estômago e intestino do paciente a fim de que este fique saciado ao se alimentar com pequena quantidade (Monteiro et al., 2019; Moura-Grec et al., 2012)

Destaca-se que a técnica de derivação gástrica-jejunal/bypass gástrico em Y-de-Roux é a mais utilizada ao promover redução do reservatório gástrico e esvaziamento pelo anel de contenção. A indicação para gastroplastia é quando o paciente tem IMC de $35-40 \mathrm{~kg} / \mathrm{m}^{2}$, associado a algum desses agravos: diabetes tipo 2, hipertensão arterial, dislidemia, apneia obstrutiva do sono, acidente vascular cerebral, ou apresentar o IMC a partir de $40 \mathrm{~kg} / \mathrm{m}^{2}$ associado a falhas nos tratamentos convencionais (Santos et al., 2019).

Embora o procedimento de cirurgia bariátrica ajude na redução do peso, sendo mais utilizada para caso de obesidade mórbida, o indivíduo pode apresentar deficiência nutricional, especialmente de cálcio, vitamina B12 e D3, ferro, proteínas e lipídeos devido à diminuição da ingestão e absorção alimentar (Cabral et al., 2016; Lefebvre et al., 2014; Vargas et al., 2020).

Posteriormente à gastroplastia, a absorção de cálcio ativo é dificultada, especialmente no jejuno e duodeno, o que repercute negativamente para as estruturas ósseas pela perda, até mesmo, no periodonto de sustentação. Quando essa absorção de cálcio é insuficiente, pode resultar em hiperparatiroidismo secundário, ou seja, elevada produção de paratormônio, o qual aumenta a atividade dos osteoclastos, resulta em destruição da cortical óssea, remoção do cálcio dos ossos e nos rins para manter sua estabilidade, gerando perda no osso da maxila e mandíbula e estreitamento da cortical óssea, aumentando suscetibilidade a fraturas, perda dentária e até mesmo do implante (Bubola et al., 2018; Cabral et al., 2016; Vargas et al., 2020)

Somando-se ainda, após à bariátrica, há menor absorção de vitamina $\mathrm{D}$, pelo jejuno e íleo, pois a mistura entre os elementos nutritivos é demorada por causa dos ácidos biliares e enzimas pancreática. Quando a vitamina D diminui, poderá acarretar na redução do cálcio dietético e calcemia, em que na tentativa de manter quantidade equilibrada de cálcio, há liberação de hormônio da paratireoide (PTH), resultando em hiperparatireodismo secundário. Então, paciente que fez cirurgia bariátrica tem risco de apresentar deficiência da vitamina D no organismo, que poderá acarretar em osteomalácia e osteoporose pela carência de cálcio, as quais irão comprometer à resistência da parte cortical e medular óssea, principalmente a densidade mineral e a qualidade do osso de todo o esqueleto (Bubola et al., 2018; Cabral et al., 2016; Moura-Grec et al., 2012).

Destaca-se que a vitamina D, o calciferol, atua no metabolismo ósseo, regulando a quantidade de cálcio e ferro no sangue. Caso haja carência de vitamina D no organismo, pode ocorrer hipocalcemia, perda de osso trabecular e comprometimento ósseo que afetarão a osseointegração. Então, quando há baixos níveis séricos de vitamina $\mathrm{D}$, o risco de perda do implante dentário é aumentado (Moura-Grec et al., 2012).

Dessa forma, posterior à gastroplastia, há risco de deficiência de vitamina $\mathrm{D}$ e minerais como o cálcio devido menor absorção no organismo, os quais são fatores que podem acarretar em falha no tratamento de implante dentário, ao interferirem no metabolismo ósseo. Destaca-se que em paciente obeso, por ter elevados níveis pró-inflamatórios, há risco de inflamação periodontal/peri-implantar que pode se acentuar após bariátrica e resultar em perda do dentária e implante (Cabral et al., 2016).

A obesidade consiste em acúmulo excessivo de tecido adiposo, sendo a sua etiologia multifatorial por envolver aspectos genéticos, psicológicos, comportamentais (alimentação e estilo de vida), sistemas nervoso, endócrino e metabólico O acúmulo exacerbado de gordura em hepatócitos e adipócitos, os quais liberam citocinas pró-inflamatórias, como interleucina 
(IL-6) e fator de necrose tumoral alfa (TNF-a), além de aumentarem a quantidade de proteína C reativa (PCR) que é um marcador inflamatório sistêmico de baixo grau, pode influenciar negativamente nas condições peri-implantares, ao aumentar a profundidade de sondagem, sangramento à sondagem e perda óssea envolto do implante pelos altos níveis inflamatórios (Alasqah et al., 2019; Cabral et al., 2016).

Dentre as formas de tratamento para obesidade, destaca-se a cirurgia bariátrica, a qual é mais utilizada em paciente com obesidade severa e que promove redução do peso corporal de forma significativa. Embora o procedimento cirúrgico de bariátrica auxilie na redução do peso devido modificações no sistema gastrintestinal, podem ocorrer: mudanças na dieta alimentar; déficit nutricional- principalmente de cálcio, ferro, vitamina D e B12- pela menor ingestão e absorção de nutrientes; problemas psicológicos como estresse, ansiedade e depressão, os quais tornam propício para que haja redução do fluxo salivar, do pH da saliva (ácido) e capacidade de tamponamento salivar, além de maior frequência de ácido gástrico na cavidade bucal por causa de regurgitação e vômito no pós-operatório (Mosquim et al., 2019; Quintella et al., 2020).

Os resultados disso, dependendo da intensidade, frequência e duração, podem ser: hipossalivação; xerostomia; halitose; erosão dentária; doenças periodontais e até mesmo hipersensibilidade, pela desmineralização de esmalte e dentina dos dentes por meio da dissolução e diminuição da capacidade de tamponamento salivar. Destaca-se que, depois da gastroplastia, distúrbios alimentares como anorexia e bulimia; hábitos parafuncionais como apertamento dentário e bruxismo; além de altas doses de medicamentos que afetam à regulação e composição salivar, aumentam a suscetibilidade de ocorrência de erosão dental (de Souza et al., 2018; Quintella et al., 2020).

Com a diminuta quantidade de 25-hidroxivitamina D (25 (OH) D) absorvida, há possibilidade de afetar o metabolismo ósseo. Já em quantidade adequada, a vitamina D influencia de forma benéfica para qualidade, volume e densidade óssea ao regular a homeostase do cálcio e do fósforo, em que, inicialmente, é formada por osteoblastos a matriz óssea orgânica e, posteriormente, dependendo da quantidade e precipitação de cálcio e fósforo, ocorre a mineralização do tecido ósseo (Cabral et al., 2016; Lefebvre et al., 2014).

A menor absorção de vitamina D e cálcio no intestino podem favorecer para o surgimento de quadro de hipocalcemia, hiperparatireodismo secundário e osteoporose e, consequentemente, interferir na osseointegração e até estabilidade do implante, por afetar estrutura cortical e medular, como na maxila que fica com alargamento das trabéculas no osso medular, porém menor espessura da cortical óssea; acarretar fraturas, reabsorções ósseas, formação excessiva de calo ósseo e cicatrização demorada (Cabral et al., 2016; Pinheiro, 2019).

Em contrapartida, a vitamina D3 ingerida e absorvida, em quantidade ideal no organismo, ajuda na inibição da produção da citocinas e da proliferação das células $T$, prevenção de doenças periodontais, defeitos ósseos e perda de inserção, por estimularem ação de monócitos/macrófagos a secretarem moléculas que têm efeitos antibióticos, além de contribuir para resposta imune, aumentar a densidade óssea peri-implantar, microarquitetura trabecular peri-implante e a fixação aprimorada do implante, favorecendo a osseointegração (Bubola et al., 2018; Quintella et al., 2020; Vargas et al., 2020).

Tendo em vista que o implante dentário é uma das formas de reabilitação oral que contribui para o retorno funcional, estético, mastigatório e até mesmo na fonação, é essencial que o implantodontista avalie minuciosamente o paciente para realizar o planejamento adequado e eficaz, especialmente em caso de indivíduo que passou pelo procedimento de cirurgia bariátrica (Cabral et al., 2016; Martins et al., 2011).

Essa avaliação inicia-se com anamnese detalhada a fim de verificar a queixa principal, histórico médico e dental, condição sistêmica, fatores de risco e alterações de saúde que possam comprometer o tratamento reabilitador como: problemas cardiovasculares, câncer, anemia, osteoporose, diabetes etc, em que ao detectar, o cirurgião-dentista direciona o paciente ao médico com finalidade de estabilizar estado de saúde e debater sobre o caso antes de possível tratamento com implante osseointegrado (Martins et al., 2011). 
Já o exame clínico permite analisar estruturas intrabucais, saúde ou infecção bucal, a qual deve ser tratada previamente, pois caso realize procedimento em paciente com periodontite não estabilizada, o quadro pode evoluir para periimplantite, especialmente em paciente obeso, e levar perda do implante (Hazem et al., 2016).

Somando-se ainda, com finalidade de avaliar indicação, diagnóstico, planejamento e risco cirúrgico, é crucial o implantodontista solicitar exames complementares, dependendo do caso, como: imaginológicos-radiográficos, hemograma, coagulograma, glicemia e entre outros. As radiografias panorâmicas e periapicais, além de tomografia computadorizada conebeam, são excelentes recursos para analisar estruturas, suporte e alterações ósseas; altura e espessura óssea; distância entre área que será colocada o implante de estruturas nobres e até mesmo verificar que, em caso de osteoporose, há menor densidade do osso e erosões na borda inferior da mandíbula podem ser vistas na radiografia (Lefebvre et al., 2014; Santos et al., 2019).

Destaca-se que, um dos elementos essenciais para o sucesso e a longevidade do tratamento de implantes dentários é a osseointegração, ou seja, união do implante ao osso alveolar receptor; porém, depende de condição de saúde geral e bucal estáveis; adequado suporte ósseo-periodontal, irrigação sanguínea suficiente e entre outros fatores. Assim, a importância da avaliação pré-operatória pelo implantodontista e seu trabalho com a equipe multidisciplinar a fim de planejamento seguro e integral (Martins et al., 2011; Santos et al., 2019; Vargas et al., 2020).

\section{Conclusão}

Portanto, a cirurgia bariátrica o paciente pode ter sua saúde geral e bucal afetadas, haja vista quadros de deficiências nutricionais pela perda de peso, redução de ingestão calórica e/ou menor absorção de nutrientes, como cálcio e vitamina D. Além de outras manifestações na cavidade oral, a deficiência nutricional como consequência da gastroplastia pode afetar negativamente o metabolismo e estrutura óssea e influenciar o tratamento com implantes dentários devido à perda óssea pela maior ação de osteoclastos e maior possibilidade de inflamação peri-implantar. No entanto, a literatura científica não apresenta evidências suficientes para afirmar que a deficiência nutricional ocasionada pela gastroplastia é capaz reduzir o potencial de osseointegração ou a longevidade dos implantes. Não obstante, são fundamentais que novos estudos clínicos sejam feitos para que possa estabelecer essa relação de forma mais esclarecedora. Assim, tendo em vista à complexidade do estado de saúde do paciente pós cirurgia bariátrica, é essencial que o implantodontista avalie, planeje e trabalhe o caso minuciosamente com equipe multidisciplinar.

\section{Referências}

Alasqah, M. N., Al-Shibani, N., Al-Aali, K. A., Qutub, O. A., Abduljabbar, T., Akram, Z. J. C. i. d., \& research, r. (2019). Clinical indices and local levels of inflammatory biomarkers in per-implant health of obese and nonobese individuals. 21(1), 80-84.

Bubola, J. P., Ferreira, R., Sant'Ana, A. C. P., de Rezende, M. L. R., Greghi, S. L. A., Damante, C. A., \& Zangrando, M. S. R. (2018). Vitamina D tem influência nos implantes dentários? J International Journal of Nutrology, 11(01), 001-010.

Cabral, J. A. V., SOUZA, G. P. d., Nascimento, J. d. A., Simoneti, L. F., Marchese, C., \& SALES-PERES, S. H. d. C. (2016). impact of vitamin D and calcium deficiency in the bones of patients undergoing bariatric surgery: a systematic review. J ABCD. Arquivos Brasileiros de Cirurgia Digestiva, 29, 120123.

Cândido, J. A. B., Moreira, M. R. C., Alexandre, S. F., Parente, N. C., \& Cavalcante, N. R. P. (2021). Obesidade em paciente com prognóstico de gravidade para Covid-19. J Research, Society and Development, 10(10), e459101019121-e459101019121.

de Souza, G. M., de Oliveira, D. W. D., Lages, F. S., Fernandes, I. A., \& Falci, S. G. M. (2018). Relationship between bariatric surgery and periodontal status: a systematic review and meta-analysis. J Surgery for obesity related diseases, 14(8), 1205-1216.

Hazem, A., Bissada, N. F., Demko, C., Paes, A., \& Lang, L. A. (2016). Comparison of Preprosthetic Implant Complications and Failures Between Obese and Nonobese Patients. J International Journal of Oral Maxillofacial Implants, 31(5).

Lefebvre, P., Letois, F., Sultan, A., Nocca, D., Mura, T., \& Galtier, F. (2014). Nutrient deficiencies in patients with obesity considering bariatric surgery: a cross-sectional study. J Surgery for obesity related diseases, 10(3), 540-546. 
Research, Society and Development, v. 11, n. 1, e48411125090, 2022

(CC BY 4.0) | ISSN 2525-3409 | DOI: http://dx.doi.org/10.33448/rsd-v11i1.25090

Martins, V., Bonilha, T., Falcón-Antenucci, R. M., Verri, A. C. G., \& Verri, F. R. (2011). Osseointegração: análise de fatores clínicos de sucesso e insucesso. $J$ Revista Odontológica de Araçatuba, 32(1), 26-31.

Monteiro, J., Pellizzer, E., Lemos, C. A., de Moraes, S., \& do Egito Vasconcelos, B. (2019). Is there an association between overweight/obesity and dental implant complications? A systematic review and meta-analysis. J International journal of oral maxillofacial surgery, 48(9), 1241-1249.

Mosquim, V., Junior, G. A. F., Hissano, W. S., Wang, L., \& Peres, S. H. d. C. S. (2019). Obesidade, cirurgia bariátrica e O impacto na saúde bucal: revisão de literatura. J. Rev. Salusvita, 117-132.

Moura-Grec, P. G., Assis, V. H., Cannabrava, V. P., Vieira, V. M., Siqueira, T. L. D., Anaguizawa, W. H., \& Sales-Peres, S. H. d. C. (2012). Consequências sistêmicas da cirurgia bariátrica e suas repercussões na saúde bucal. J ABCD. Arquivos Brasileiros de Cirurgia Digestiva, 25, 173-177.

Munzlinger, E., Narcizo, F. B., \& de Queiroz, J. E. R. (2012). Sistematização de revisões bibliográficas em pesquisas da área de IHC. Paper presented at the IHC (Companion).

Pinheiro, F. P. (2019). Avaliação histomorfométrica da osseointegração de implantes em pacientes com alterações metabológicas.

Quintella, M. C. M., Farias, T. M. C. P., SoutoMaior, J. R., da Silva Casado, B. G., de Souza Leão, R., \& de Moraes, S. L. D. (2020). Relationship between bariatric surgery and dental erosion: a systematic review. J Surgery for Obesity Related Diseases 16(9), 1283-1290.

Santos, L. R. A. C., Nobre, L. B., Silva, R. N., Nóbrega, D. F., de Albuquerque, S. A. V., \& dos Santos, N. B. (2019). Cirurgia bariátrica e suas repercussões na saúde bucal: uma revisão de literatura. J Diversitas Journal, 4(2), 612-621.

Vargas, J. A., Bonato, R. C. S., Orenha, E. S., \& Sales, S. H. d. C. (2020). Avaliação Do Padrão Ósseo Alveolar Em Mulheres Obesas E Não Obesas, Antes E Após A Cirurgia Bariátrica: Um Estudo De Coorte Prospectivo. JABCD. Arquivos Brasileiros de Cirurgia Digestiva., 33. 